\title{
METABOLIC PIGMENTARY RETINOPATHIES
}

The diagnosis and management of inborn errors of metabolism associated with retinal degeneration are reviewed from the Clinique et Unite de Recherche de Génétique Médicale, the Hopital des Enfants Malades and the Opthalmologic Department et Formation Associée de Recherches Claude Bernard, Hopital Necker - Enfants-Malades - Laennec, Paris, France. Pigmented retinopathies are divided into two groups: (1) primary RP confined to the eyes; and (2) secondary RP associated with single or multiple organ system disease. Secondary $R P$ is most often associated with nervous system involvement, morphological abnormalities, deafness, myopathy, nephropathy, and skin abnormalities. Some are well defined non-metabolic genetic syndromes and others are related to inherited metabolic disorders. In a patient presenting with retinal abnormalities the search for a metabolic disorder will concentrate on (1) errors of lipid metabolism; (2) errors of peroxisomal functions; and (3) errors of mitochondrial functions including the respiratory chain. Biochemical investigation should be considered in patients with ocular deficits that are associated with the following abnormalities: (1) neurological involvement including muscle hypotonia, seizures, peripheral neuropathies, cerebellar signs, sensorineural hearing deficit, cerebral abnormalities, mental retardation; (2) craniofacial abnormalities; (3) hepatological abnormalities; (4) renal abnormalities; (5) cardiomegaly; (6) skeletal abnormalities; (7) skin abnormalities; (8) failure to thrive; (9) hormonal disturbances; and (10) progression of symptoms. The screening procedure for metabolic disorders includes (1) peripheral blood for acanthocytes; (2) lipid pattern including lipoproteins; (3) redox status including lactic acid, pyruvate and ketones; (4) amino acid pattern; (5) organic acid pattern in the urine; (6) very long chain fatty acids and phytanic acid; and (7) enzymatic studies (Poll-The BT et al. Metabolic pigmentary retinopathies: diagnosis and therapeutic attempts. Euro J Pediatr Jan 1992;

151:2-11). (Reprints: Dr. B.T. Poll-The, Children's Hospital, "Wilhelmina Kinderziekenhuis" Metabolic Department, Nieuwe Gracht 137, NL-3512 LK Utrecht, The Netherlands.)

COMMENT. This excellent review of the metabolic retinal degenerations includes case reports of the major errors of metabolism associated with retinal degeneration including abetalipoproteinemia, neuronal ceroid lipofuscinosis, classical and infantile Refsum diseases, hydroxydicarboxylic aciduria, Sjögren-Larsson syndrome, Kearns-Sayre syndrome and peroxisomal disorders (see Progress in Pediatric Neurology, Millichap JG ed. 1991 pp. 470-474).

\section{MITOCHONDRIAL CYTOPATHIES}

\section{MITOCHONDRIAL MYOPATHY WITH DNA DEPLETION}

Five children with mitochondrial myopathy associated with depletion of muscle mtDNA are reported from the Departments of Neurology and Genetics and Development, Columbia University College of Physicians and Surgeons, 